\title{
High resolution analysis of the intracellular proteome of cervical cancer cell lines unveils novel regulators of cervical carcinogenesis
}

\author{
KALLIOPI I. PAPPA ${ }^{1,2}$, VASILIKI LYGIROU ${ }^{3,4}$, GEORGIA KONTOSTATHI ${ }^{3,4}$, \\ MANOUSOS MAKRIDAKIS ${ }^{3}$, KONSTANTINOS VOUGAS ${ }^{3}$, ANTONIOS SFAKIANAKIS ${ }^{2}$, \\ GEORGE DASKALAKIS $^{2}$, JEROME ZOIDAKIS ${ }^{3}$ and NICHOLAS P. ANAGNOU ${ }^{1,4}$
}

${ }^{1}$ Cell and Gene Therapy Laboratory, Centre of Basic Research II, Biomedical Research Foundation of The Academy of Athens (BRFAA), 11527 Athens; ${ }^{2}$ The First Department of Obstetrics and Gynecology, Medical School, National and Kapodistrian University of Athens, Alexandra Hospital, 11528 Athens; ${ }^{3}$ Biotechnology Division, Centre of Basic Research, Biomedical Research Foundation of The Academy of Athens (BRFAA); ${ }^{4}$ Laboratory of Biology, Medical School, National and Kapodistrian University of Athens, 11527 Athens, Greece

Received November 2, 2018; Accepted April 16, 2019

DOI: $10.3892 /$ or.2019.7269

\begin{abstract}
Cervical cancer remains the fourth most common and most lethal type of cancer in women, despite the applied regular screening and prevention strategies, while the available treatment schemes still pose a threat to fertility. Substantial understanding of the underlying mechanisms and development of novel diagnostic, prognostic and therapeutic approaches are critical steps for improving cervical cancer management. Towards this goal, a comparative proteomic analysis was conducted between three cervical cancer cell lines (HeLa: HPV18 ${ }^{+}$, SiHa: HPV16 ${ }^{+}$, C33A: HPV') and normal cervical keratinocytes (HCK1T). The total cell extract of each cell line was analyzed by liquid chromatography coupled to tandem mass spectrometry (LC-MS/MS). Differential expression analysis revealed 919,826 and 1,370 deregulated proteins in the comparisons of HeLa, SiHa and C33A with HCK1T cell lines, respectively. Pathway enrichment analysis of the differentially expressed proteins highlighted common cancer characteristics such as high metabolic demands and increased cell turnover, confirming the validity of the proteomic results. Extensive literature mining of the consistently differentially expressed proteins that resulted from the three comparisons was performed leading to a shortlist of 21 proteins that are potentially involved in cervical malignancy. The criteria for this shortlisting were the association of the proteins with various types of cancer, while there is no study as yet associating their
\end{abstract}

Correspondence to: Dr Kalliopi I. Pappa, The First Department of Obstetrics and Gynecology, Medical School, National and Kapodistrian University of Athens, Alexandra Hospital, 80 Vasilissis Sofias Avenue, 11528 Athens, Greece

E-mail: kalliopi.pappa20@gmail.com

Key words: cervical cancer, proteomics, LC/MS-MS, cervical cell lines expression to cervical cancer. Moreover, the expression trend of two of the shortlisted proteins was validated using western blot analysis. The proteomic datasets generated in this study can be utilized to enrich the current knowledge on cervical cancer pathology and unveil key molecular mechanisms of carcinogenesis. In conclusion, the shortlist of consistently deregulated proteins between cervical cancer cell lines and normal cervical keratinocytes can be used for validation in clinical samples and in functional investigation experiments that could ultimately lead to the discovery of novel disease biomarkers and drug targets.

\section{Introduction}

Cervical cancer remains the fourth most common and the fourth most fatal type of cancer in women. Based on GLOBOCAN estimates, there will be a total of 569,847 new cases of cervical cancer and 311,365 deaths due to cervical cancer in 2018 worldwide (1). The main underlying cause of cervical cancer is human papillomavirus (HPV) infection. HPV infections may cause mild dysplasia which usually regresses spontaneously but some infections caused by high-risk HPV types, may become persistent and progress to high grade lesions and eventually invasive carcinoma (2).

Although cervical cancer is not easily detected and treatable, the long window of time between precancerous lesions and invasive cancer provides the opportunity for early detection and prevention. Indeed, regular cytology-based screening has significantly reduced but not eliminated cervical cancer mortality (3), while the most recent advances concerning HPV testing and HPV vaccines have further improved the disease control, mainly in high-income countries (4). However, a significant number of new cases are still diagnosed.

The treatment of cervical cancer relies on surgical interventions that are usually accompanied by chemotherapy and radiation. Elimination of the cancer and patient survival are the top priorities; however, since childbirth is often delayed in developed countries, preservation of fertility is a major 
factor when considering cervical cancer treatment approaches. Fertility sparing techniques have been developed but they can only be applied after careful evaluation of the cancer and in early stages $(5,6)$. Targeted pharmaceutical treatment approaches could be the solution to this problem, but despite the intensive efforts during the last decades, no novel drugs have been applied in clinical practice $(7,8)$.

A need for deeper understanding of the underlying mechanisms of cervical cancer pathology is critical. Towards this goal, our group has already investigated the secretome (9) and the membrane proteomes (10) of three cervical cancer cell lines in comparison to normal cervical keratinocytes with liquid chromatography coupled with tandem mass spectrometry (LC-MS/MS). These studies highlighted the inhibition of matrix metalloproteases (9) and the deregulation of HIPPO, PI3K/Akt, and EIF2 signaling pathways (10) in cervical cancer as well as many differentially expressed proteins that are potentially crucial for cervical malignancy. Along the same lines and in an attempt to elucidate the molecular mechanisms underlining cervical carcinogenesis, we analyzed the intracellular proteome of three cervical cancer cell lines against normal cervical keratinocytes employing high resolution LC-MS/MS. The differentially expressed proteins between the cervical cancer cell lines and the control cell line were integrated into molecular pathways that are possibly deregulated during the malignant transformation of the cervical epithelium. Through an extensive review of the existing literature, we shortlisted the consistent proteomic changes providing a valuable dataset of proteins for further investigation and functional analyses. These proteins could be used as molecular markers or drug targets

\section{Materials and methods}

Cell culture. HeLa (HPV 18+), SiHa (HPV 16 ${ }^{+}$) and C33A $\left(\mathrm{HPV}^{-}\right)$cervical cancer cell lines, were purchased from the American Type Culture Collection (ATCC) (Manassas, VA, USA) and cultured in Dulbecco's modified Eagle's medium (DMEM) supplemented with $10 \%$ fetal bovine serum (FBS) (Gibco/Invitrogen; Thermo Fisher Scientific, Inc., Waltham, MA, USA) and $1 \%$ penicillin-streptomycin at $37^{\circ} \mathrm{C}$ in $5 \% \mathrm{CO}_{2}$ as previously described (11). HCK1T cells were a kind gift from Dr Tohru Kiyono of the National Cancer Center Research Institute (Chuo-ku, Tokyo, Japan) (12) and were cultured as proposed (13) in Defined Keratinocyte Serum-Free Medium (SFM) (Gibco; Thermo Fisher Scientific, Inc.) supplemented with $5 \mathrm{ng} / \mathrm{ml}$ epidermal growth factor (EGF) (Gibco; Thermo Fisher Scientific, Inc.) and $50 \mu \mathrm{g} / \mathrm{ml}$ of bovine pituitary extract (BPE) (Gibco; Thermo Fisher Scientific, Inc.). When the cells reached a concentration of $10^{6}$ cells per $\mathrm{ml}$, they were trypsinized, harvested, and the pellets were washed in $1 \mathrm{X}$ phosphate-buffered saline (PBS) 3 times before storage at $-80^{\circ} \mathrm{C}$ until further use.

Sample preparation. Pellets were homogenized in homogenization buffer (7 M urea, $2 \mathrm{M}$ thiourea, 4\% CHAPS, $1 \%$ DTE) using mild sonication (water bath sonication). After centrifugation at $16,000 \times \mathrm{g}$ for $20 \mathrm{~min}$, the total cell extract was obtained as a supernatant. Protein concentration was measured with the Bradford assay. The sample preparation was based on the GeLC-MS protocol described by Makridakis and Vlahou (14). Briefly, $10 \mu \mathrm{g}$ of protein extract from each sample were analyzed by $12 \%$ SDS PAGE and stained with Coomassie Colloidal Blue (Fluka; Thermo Fisher Scientific, Inc.) overnight. The gel bands were excised and cut in small pieces (1-2 mm side squares). Gel pieces were destained in $40 \%$ acetonitrile (Thermo Fisher Scientific, Inc.), $50 \mathrm{mM} \mathrm{NH} \mathrm{HCO}_{3}$ (Sigma-Aldrich; Merck KGaA, Darmstadt, Germany), reduced in $10 \mathrm{mM}$ dithioerythritol (Sigma-Aldrich;

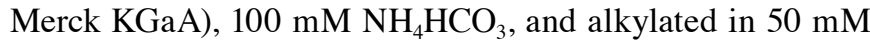
iodoacetamide (Applichem, Inc., Omaha, NE, USA), $100 \mathrm{mM}$ $\mathrm{NH}_{4} \mathrm{HCO}_{3}$. Samples were dried using the Savant Speedvac ${ }^{\mathrm{TM}}$ concentrator (Thermo Fisher Scientific, Inc.) and trypsinized overnight at room temperature with $600 \mathrm{ng}$ trypsin (Roche Diagnostics, Basel, Switzerland) in $10 \mathrm{mM} \mathrm{NH}_{4} \mathrm{HCO}_{3}$. Peptide extraction was performed with a wash of the trypsinized gel pieces with $50 \mathrm{mM} \mathrm{NH}_{4} \mathrm{HCO}_{3}$, followed by two washes with $50 \%$ acetonitrile (Thermo Fisher Scientific, Inc.), 5\% formic acid (Sigma-Aldrich; Merck KGaA) for $15 \mathrm{~min}$ at room temperature, under shaking conditions. Extracted peptides were dried using the Savant Speedvac ${ }^{\mathrm{TM}}$ concentrator and analyzed by LC-MS/MS.

LC-MS/MS (liquid chromatography coupled with tandem mass spectrometry) analysis. Analysis of the protein extracts was conducted with an UltiMate 3000 Nano HPLC Dionex Ultimate $^{\circledR} 3000$ RSLS system (Dionex $^{\text {TM }}$; Thermo Fisher Scientific, Inc.) as previously reported (9). Dried peptides were solubilized in $10 \mu \mathrm{l}$ mobile phase $\mathrm{A}(0.1 \%$ formic acid $)$ and $5 \mu \mathrm{l}$ from each sample was loaded on a $0.1 \times 20 \mathrm{~mm}, 5-\mu \mathrm{m}$ C18 nanotrap column. The loading was performed at a flow rate of $5 \mu \mathrm{l} / \mathrm{min}$ in $98 \%$ mobile phase A [0.1\% formic acid (Sigma-Aldrich; Merck $\mathrm{KGaA}$ )] and 2\% mobile phase $\mathrm{B}$ [100\% acetonitrile (Thermo Fisher Scientific, Inc.)], 0.1\% formic acid. Then, the sample was analyzed using an Acclaim PepMap C18 nanocolumn $75 \mu \mathrm{m}$ x $50 \mathrm{~cm}$ (Dionex $^{\mathrm{TM}}$; Thermo Fisher Scientific, Inc), at a flow rate of $0.3 \mu \mathrm{l} / \mathrm{min}$. The trap and the nanoflow column were maintained at $35^{\circ} \mathrm{C}$. The samples were eluted with a gradient of solvent $\mathrm{B}$, starting at $1 \% \mathrm{~B}$ for $5 \mathrm{~min}$, rising to $5 \% \mathrm{~B}$ at $10 \mathrm{~min}, 25 \% \mathrm{~B}$ at $180 \mathrm{~min}$ and $65 \% \mathrm{~B}$ at $240 \mathrm{~min}$. The column was then washed and re-equilibrated prior to injection of the next sample. The eluent was ionized using a Proxeon Nano Spray Electron Spray Ionization (ESI) source, operating in positive ion mode into an Orbitrap Elite FTMS (Thermo Fisher Scientific, Inc). Ionization voltage was at $2.6 \mathrm{kV}$ and the capillary temperature was at $200^{\circ} \mathrm{C}$. The mass spectrometer was operated using the MS/MS mode scanning from 380 to 2,000 amu (atomic mass units). The resolution of ions in MS1 was 60,000, and 15,000 for HCD MS2. The top 20 multiply-charged ions were selected from each scan for MS/MS analysis using HCD at $35 \%$ collision energy. Dynamic exclusion was set to $30 \mathrm{sec}$.

MS data analysis. Peptide identification was performed with Proteome Discoverer 1.4 software package (Thermo Scientific, Inc.), using the SEQUEST search engine and the UniProt (http://www.uniprot.org/) human reviewed database, updated on May 30, 2016, including 20,204 entries. The search was performed using carbamidomethylation of cysteine as static, and oxidation of methionine as dynamic modifications. Two 
missed cleavage sites, a precursor mass tolerance of $10 \mathrm{ppm}$ and fragment mass tolerance of 0.05 Da were allowed. False discovery rate (FDR) validation was based on q value: target FDR (strict), 0.01; target FDR (relaxed), 0.05. SEQUEST results were filtered for false-positive identifications. Peptide quantification was performed as previously described (15). Only peptides being present in $75 \%$ of the samples in at least one group (cervical cancer or normal cell line) were considered confident identifications and were used for protein quantification. Statistical analysis was based on the Mann-Whitney test performed in R (version 3.3.1). Differences with P-value $\leq 0.05$ were considered statistically significant and an expression change threshold of 2 (cancer/normal $\geq 2$ or cancer/normal $\leq 0.5$ ) was also applied for differential expression. The protein expression heatmap was created at http://www.heatmapper.ca/ (16).

Functional analysis. Functional analysis was performed with the ClueGO plug-in (17) in Cytoscape 3.4 .0 (18). Ontologies were retrieved from REACTOME pathways database (updated on September 4, 2017) and only statistically significant pathways (Bonferroni corrected P-value $\leq 0.05$, two-sided hypergeometric test) were taken into account. For the remaining parameters, default settings were used. Results were simplified based on biological relevance and only the leading term from each group is presented.

Western blot analysis. Four 30- $\mu \mathrm{g}$ samples of cell extract dissolved in Laemli's buffer from each cell line were loaded on $10 \%$ SDS-polyacrylamide gel after incubation at $90^{\circ} \mathrm{C}$ for $10 \mathrm{~min}(2 \mathrm{gels}$ in total with 2 replicates from each cell line on each gel). The gel was run at $40 \mathrm{~V}$ for $15 \mathrm{~min}$ and then at $120 \mathrm{~V}$ in Tris-glycine-SDS buffer. The transfer was performed in transfer buffer (3.03 $\mathrm{g}$ Tris, $14.4 \mathrm{~g}$ glycine, $200 \mathrm{ml}$ methanol for 1 liter total volume) for $2 \mathrm{~h}$ at $290 \mathrm{~mA}$ at $4^{\circ} \mathrm{C}$. Then, the membrane was stained with Ponceau-S stain for $5 \mathrm{~min}$, washed with ultrapure water for $5 \mathrm{~min}$ three times, followed by the addition of blocking solution $(5 \% \mathrm{w} / \mathrm{v}$ non-fat dried milk in TBS-Tween $0.1 \% \mathrm{v} / \mathrm{v}$ ) and incubation for $2 \mathrm{~h}$. The membrane was washed with TBS-Tween $0.1 \% \mathrm{v} / \mathrm{v}$ successively for 15,5 and $5 \mathrm{~min}$, and the primary antibodies: LIMA1 (sc-136399; dilution, 1:200; Santa Cruz Biotechnology, Inc., Dallas, TX, USA), PON2 (sc-373981; dilution, 1:100; Santa Cruz Biotechnology, Inc., Dallas, TX, USA) and $\alpha$-tubulin (T6199; dilution, 1:5000; Sigma-Aldrich; Merck $\mathrm{KGaA}$ ) were added at the respective dilutions and left at $4^{\circ} \mathrm{C}$ overnight. The next day, the three washes were repeated and the secondary goat anti-mouse antibody IgG-HRP (sc-2005; Santa Cruz Biotechnology, Inc.) was added at a 1:2,000 dilution and left at room temperature for $2 \mathrm{~h}$. The three washes were repeated, ECL was added and left for $1 \mathrm{~min}$; its excess was removed, followed by film exposure and development. The films were scanned and the quantification of the bands was performed with Quantity One software 4.4.1 (Bio-Rad Laboratories, Hercules, CA, USA). For LIMA1, the bands corresponding to 110 and $90 \mathrm{kDa}$ were quantified. For PON2, the bands corresponding to 53 and $43 \mathrm{kDa}$ were quantified. Both antibodies (LIMA1 and PON2) were blotted on the same membranes (since the difference in molecular weights allowed this) and quantification of $\alpha$-tubulin was used as a loading control for both antibodies. One-way ANOVA and Tukey HSD tests were performed in SPSS Statistics 22.0 (IBM, Armonk, NY, USA).

\section{Results}

Differential expression analysis. Towards a better understanding of the cervical cancer pathology and the molecular mechanisms underlying the malignant transformation of the cervical epithelium, a comparative proteomic analysis of cervical cell lines was performed, utilizing the GeLC-MS protocol (14). The cell lines that were analyzed included the following: HCK1T (human cervical keratinocytes), a normal cervical epithelium cell line; HeLa, a cervical cancer cell line positive for HPV18 (HPV18 $)$; SiHa, a cervical cancer cell line positive for HPV16 (HPV16 ${ }^{+}$); and C33A, a cervical cancer cell line negative for HPV (HPV'). Four biological replicates were processed from each cell line. A graphical representation of our workflow is presented in Fig. 1.

The total number of protein identifications was 2,607 for HCK1T; 2,859 for HeLa; 2,902 for SiHa; and 3,405 for C33A. Each of the three cancer cell lines (HeLa, SiHa, C33A) was compared to the normal cell line (HCK1T) and only peptides detected in at least $75 \%$ of the samples (3 out of 4 biological replicates) in at least one group (cervical cancer or normal cell line) were taken into account for the analysis. The differentially expressed proteins in cancer vs. normal cells were considered the proteins that showed a statistically significant change (Mann-Whitney P-value $\leq 0.05$ ) and had an expression change of at least 2 (cancer/normal $\geq 2$ or cancer/normal $\leq 0.5$ ). In addition, proteins uniquely identified only in one cell line per comparison were also considered as differentially expressed proteins.

In the comparison between the HeLa and HCK1T cells, 919 proteins were differentially expressed. Out of these, 321 were upregulated and 165 were downregulated in cancer, while 257 proteins were uniquely identified in HeLa and 176 proteins were uniquely identified in HCK1T cells (Supplementary Table I, available at Figshare, doi: 10.6084/m9.figshare.7121540; https://figshare.com/articles/Supplementary_data/7121540).When comparing SiHa to HCK1T cells, 826 proteins were differentially expressed in total, out of which 310 were upregulated in cancer, 138 were downregulated in cancer, 224 proteins were detected only in $\mathrm{SiHa}$ and 154 proteins were only detected only in HCK1T (Supplementary Table II, available at Figshare, doi: 10.6084/m9.figshare.7121540; https:/figshare.com/articles/Supplementary_data/7121540). Finally, in regards to the comparison of C33A to HCK1T cells, the total number of differentially expressed proteins was 1,370. Out of these proteins, 497 were upregulated in cancer, 174 were downregulated in cancer, 472 were identified only in C33A cells and 227 proteins were only identified in HCK1T cells (Supplementary Table III, available at Figshare, doi: 10.6084/m9.figshare.7121540; https://figshare. com/articles/Supplementary_data/7121540).

Functional analysis. Functional annotation and pathway enrichment of the differentially expressed proteins per comparison was performed using the ClueGO plug-in of Cytoscape. The functional analysis results for the comparisons HeLa vs. HCK1T, SiHa vs. HCK1T, and C33A vs. HCK1T are presented in 

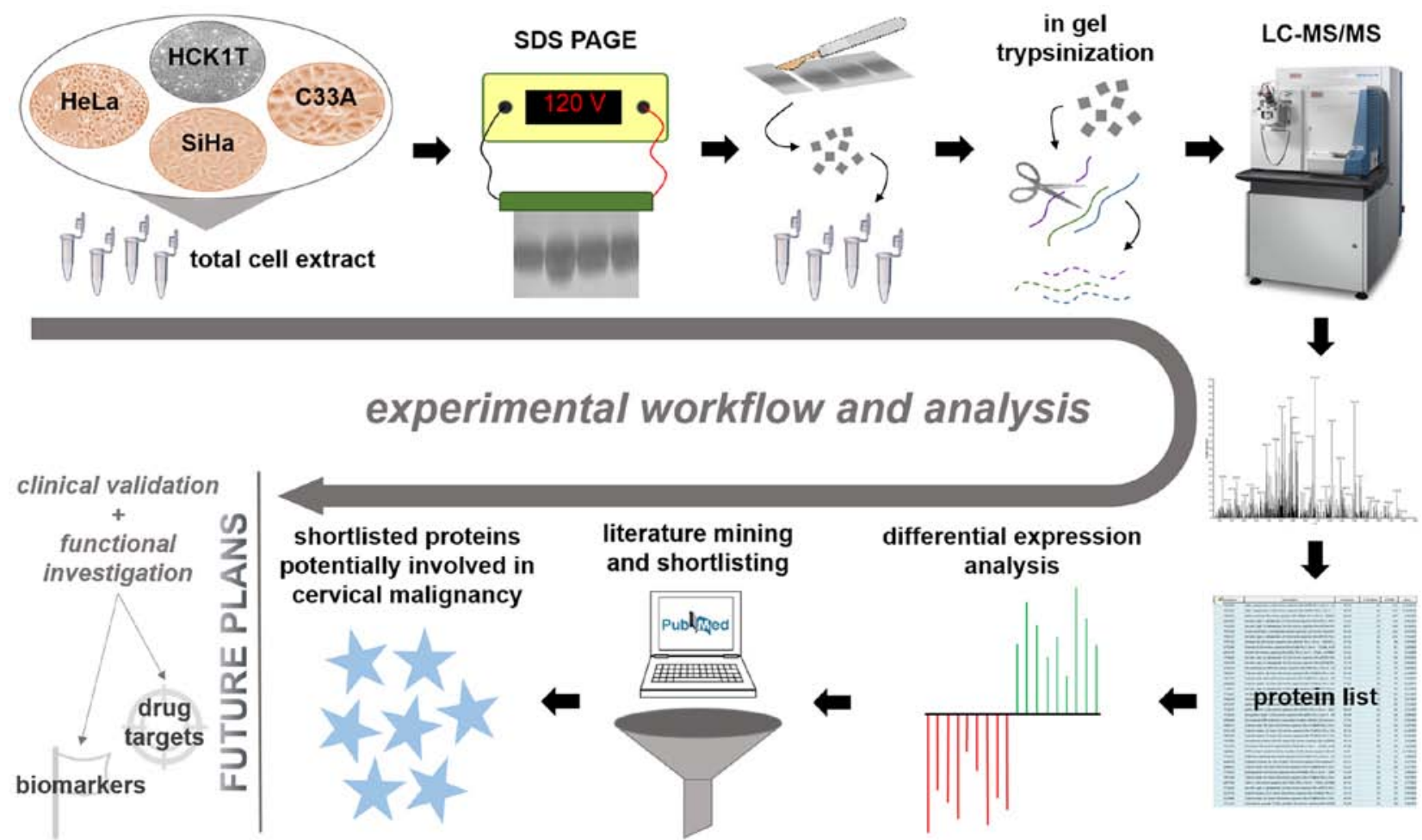

Figure 1. Graphical representation of the experimental workflow and analysis followed. The total cell extract from three cervical cancer cell lines [HeLa $\left(\mathrm{HPV}_{1}^{+}\right)$, SiHa $\left.\left(\mathrm{HPV}^{+} 6^{+}\right), \mathrm{C} 33 \mathrm{~A}\left(\mathrm{HPV}^{-}\right)\right]$and normal cervical keratinocytes (HCK1T) was collected and analyzed by SDS-PAGE, following the GeLC-MS/MS protocol. The bands were excised from the gel and cut into small pieces where in-gel trypsinization was performed. The extracted peptides were then analyzed by LC-MS/MS and the resulting spectra were translated into protein lists. After differential expression analysis, an extensive literature mining was performed in order to shortlist the proteins based on existing studies on cancer and cervical cancer, in particular. The datasets and shortlisted proteins from this study can be used for clinical validation (e.g. immunohistochemistry, multiple reaction monitoring mass spectrometry) and functional investigation with the aim to develop novel biomarkers and drug targets. LC-MS/MS, liquid chromatography-tandem mass spectrometry.

Figs. 2-4, respectively, and Supplementary Table IV (available at Figshare, doi: 10.6084/m9.figshare.7121540; https://figshare. com/articles/Supplementary_data/7121540). Some of the prominent and significant pathways that appear to be deregulated between the cervical cancer and the normal cell lines are metabolism of proteins, metabolism of nucleotides, unwinding of DNA, glycolysis, gluconeogenesis apoptosis and oxidative stress induced senescence. These pathways reflect the malignant phenotype of the cancer cells with high metabolic requirements, increased cell turnover and damage control mechanisms, as expected.

Focusing on the consistent proteomic changes. In order to focus on the common most prominent proteomic changes between cervical cancer and normal cervical keratinocytes, regardless of the cancer origin (presence of HPV infection, HPV type), a shortlisting of the differentially expressed proteins that emerged from the three comparisons (HeLa vs. HCK1T, SiHa vs. HCK1T and C33A vs. HCK1T) was performed. As a first step, only proteins that followed the same expression trend in cancer vs. normal cells were selected. Proteins that were uniquely identified in only one cell line per comparison were excluded at this point due to probable technical limitations such as protein quantity being below the limit of detection of the method used but not completely absent, and challenging reproducibility, i.e. its absence might not be confirmed with a different method. This first shortlisting resulted in 105 consistent proteomic changes between the cervical cancer cell lines and the normal cervical keratinocytes, which are represented by a heatmap in Fig. 5. Following this, an extensive literature mining for all the consistently differentially expressed proteins was performed (Supplementary Table V, available at Figshare, doi: 10.6084/m9.figshare.7121540; https://figshare. com/articles/Supplementary_data/7121540). The aim of this bibliographic investigation was to reveal proteins that are potential key regulators of cervical carcinogenesis but have not yet been investigated. This search led to a list of 21 proteins (Table I) that are implicated in cancer but there is no study associating their expression to cervical cancer, at least to our knowledge, and could be the basis of further investigations in the context of cervical cancer.

Validation of the expression trend for two of the shortlisted proteins. LIM domain and actin-binding protein 1 (LIMA1) and serum paraoxonase/arylesterase 2 (PON2) were also assessed with western blot analysis in four new biological replicates from each of the four cervical cell lines. Their expression trend based on western blot analysis was similar to the proteomics results, as shown in Fig. 6, validating the credibility of the method. Specifically, both isoforms of LIMA1 (bands of 90 and $110 \mathrm{kDa}$ in the western blot analysis) are shown to be downregulated in the cervical cancer cell lines compared to HCK1T, according to both methodologies. In a similar way, PON2 is shown to be upregulated in all cervical cancer cell lines, by both approaches [the double band of PON2 in the Western blot is a common observation (19)]. 


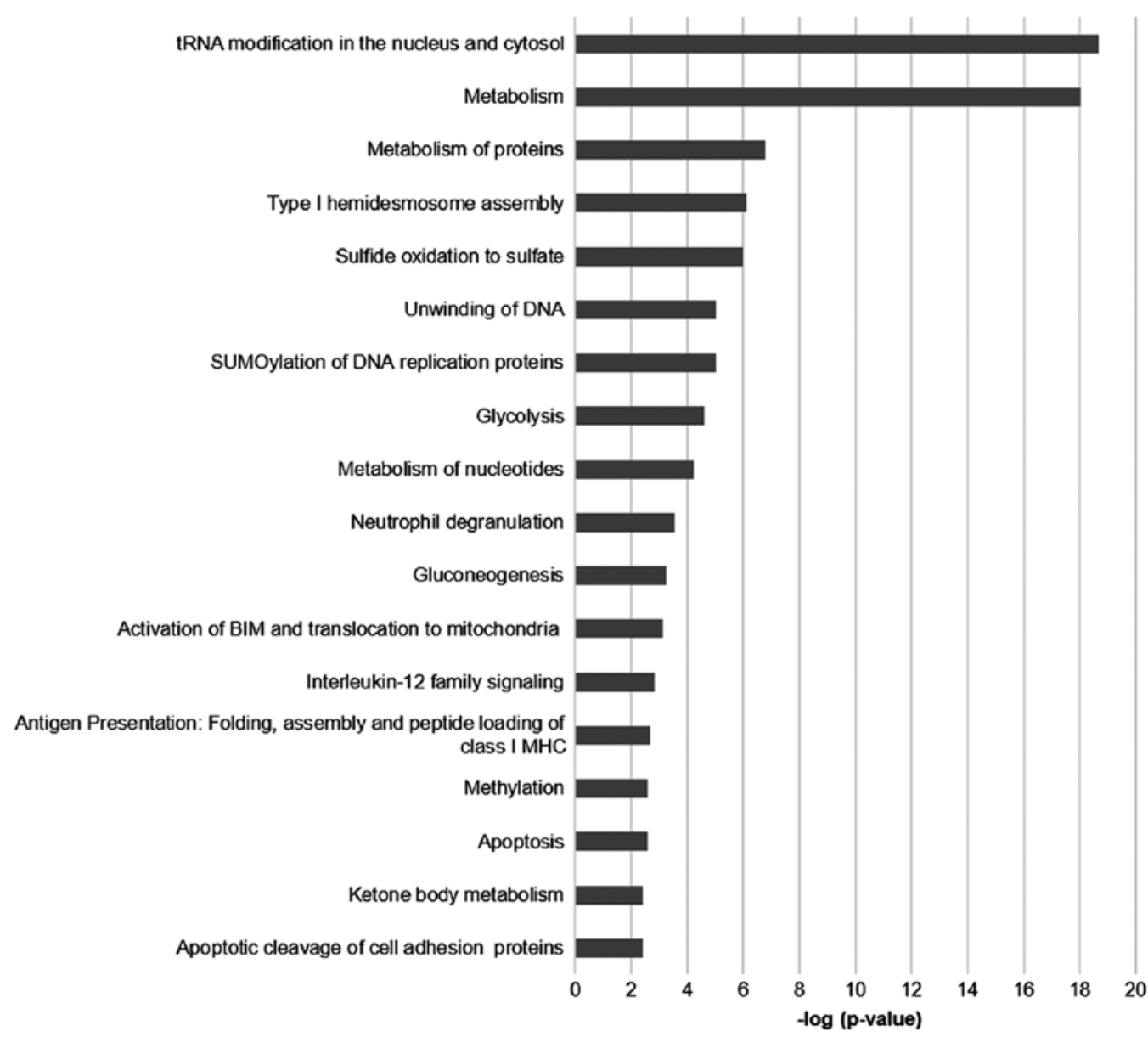

Figure 2. Reactome pathways enriched by the differentially expressed proteins between the HeLa and HCK1T cell lines. Results were simplified based on biological relevance and only the leading term from each group is presented (Bonferroni corrected P-value $\leq 0.05$, two-sided hypergeometric test).

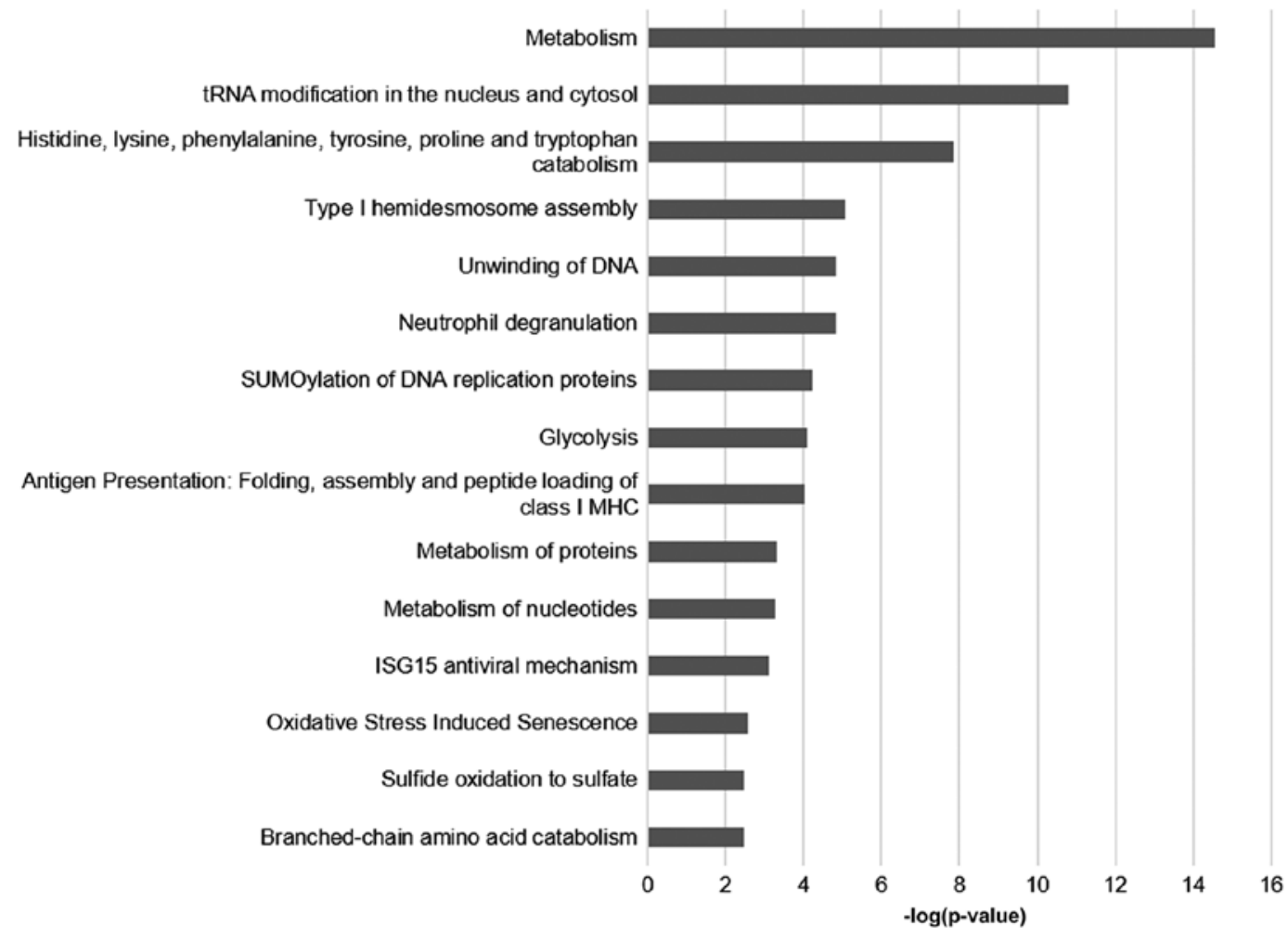

Figure 3. Reactome pathways enriched by the differentially expressed proteins between the SiHa and HCK1T cell lines. Results were simplified based on biological relevance and only the leading term from each group is presented (Bonferroni corrected P-value $\leq 0.05$, two-sided hypergeometric test). 


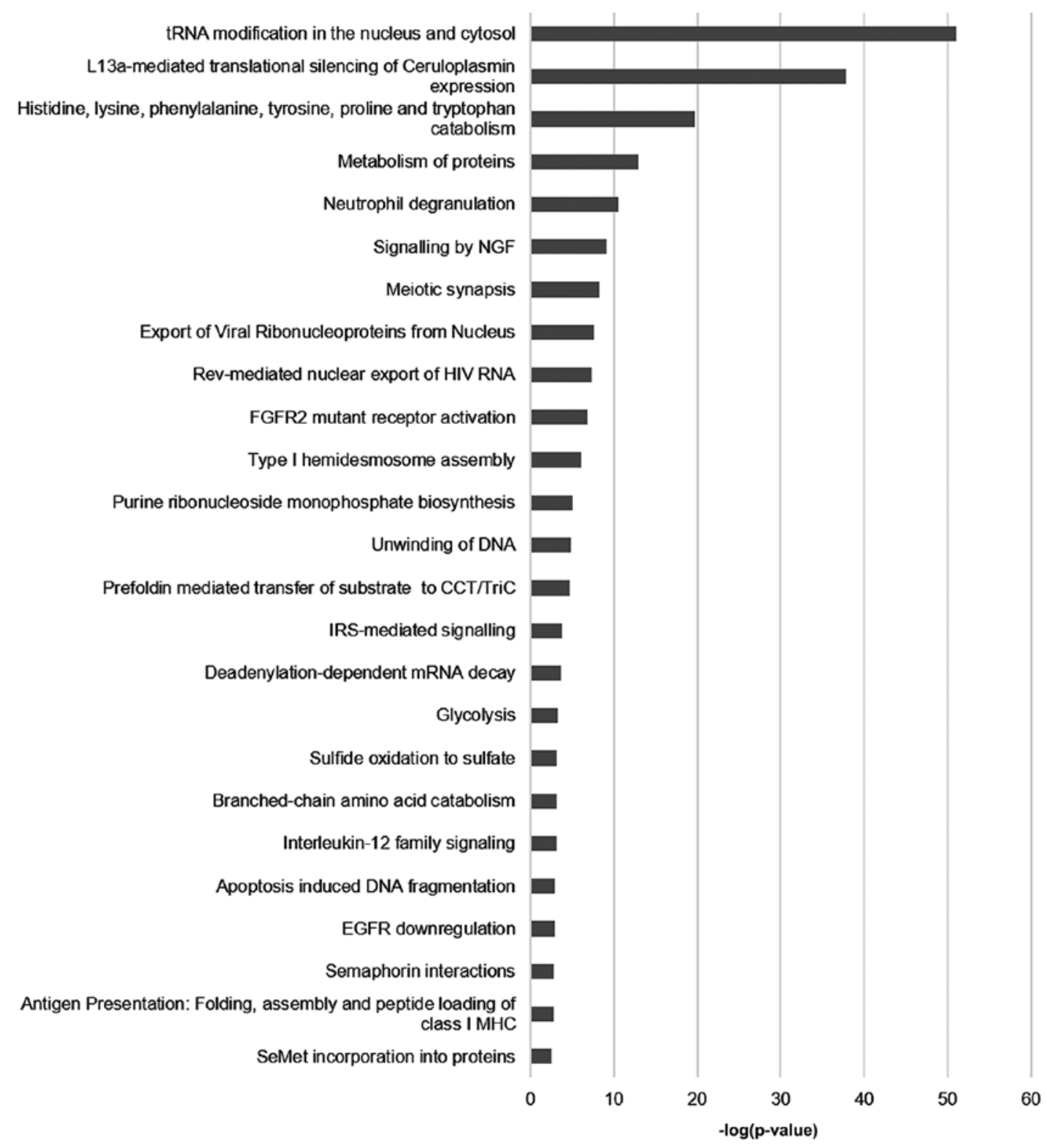

Figure 4. Reactome pathways enriched by the differentially expressed proteins between the C33A and HCK1T cell lines. Results were simplified based on biological relevance and only the leading term from each group is presented (Bonferroni corrected P-value $\leq 0.05$, two-sided hypergeometric test).

\section{Discussion}

The comprehensive proteomic analysis of cervical cancer cell lines and normal cervical keratinocytes, performed utilizing the LC-MS/MS platform, revealed the proteomic changes occurring during the malignant transformation of the cervical epithelium in three subtypes of cervical cancer: HPV $18^{+}$ (HeLa cells), HPV16 ${ }^{+}$(SiHa cells) and HPV ${ }^{-}$(C33A cells). The cell lines analyzed were chosen carefully for this study, as they represent the most common high-risk HPV-infected cervical cancers and HPV-free cervical cancer that, together, constitute a great percentage of the cervical cancer incidents. The cancer cell lines were compared to normal cervical keratinocytes and not keratinocytes of another origin (e.g. skin) to eliminate differences due to tissue-specific characteristics.

The differentially expressed proteins in each comparison (HeLa vs. HCK1T, SiHa vs. HCK1T, and C33A vs. HCK1T) can be utilized for further analysis and can be the basis for systems biology approaches. The pathway enrichment analysis of the differentially expressed proteins from each comparison revealed that the deregulation processes are associated with the elevated metabolic demands and increased cell turnover that are common characteristics of cancer (20). These findings confirm the biological relevance of our proteomics results and add to the integrity of the study. Furthermore, the shortlisting of the differentially expressed proteins performed based on the existing literature, paves the way for further investigation of promising candidates, clinical validation and functional exploration in the context of cervical cancer. Validation of the expression trend of two of the shortlisted proteins using an immuno-based technique (western blot analysis) further confirmed the robustness and accuracy of the proteomics methodology.

The 21 shortlisted proteins (Table I) have been associated with other types of cancer in the literature, such as breast, prostate, ovarian and lung cancer, but have not yet been studied within the context of cervical cancer, as far as we know. These 


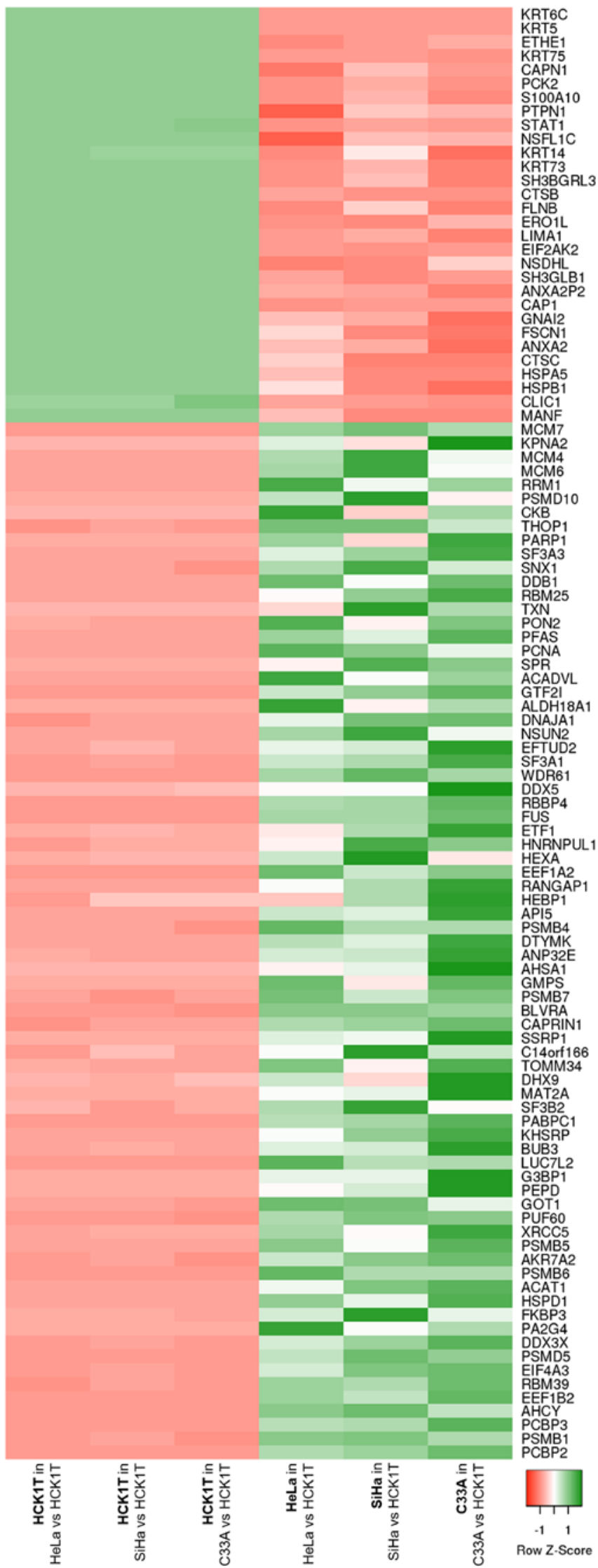

Figure 5. Expression heatmap of the 105 consistent proteomic changes between the cervical cancer lines and the normal cervical keratinocytes. Levels of expression are represented with color intensity (green for high and red for low) for each cell line. Protein levels noted in the HCK1T cell line exhibited variations when compared to each of the three cervical cancer cell lines due to the peptide frequency threshold applied (see Materials and methods). proteins could be of great interest for further investigations and could potentially play pivotal roles in cervical carcinogenesis. Few of the proteins with functional relevance to cancer are discussed below.

LIM domain and actin-binding protein 1 (LIMA1), which is involved in the regulation of actin dynamics, has been found to be downregulated in various cancer types when compared to healthy tissue (21). In the present study, LIMA1 was found to be consistently downregulated in all three cervical cancer cell lines compared to HCK1T cells in the proteomic analysis. This downregulation was also confirmed using western blot analysis. In the literature, it has been shown that LIMA1 expression significantly decreases concomitantly with cancer progression and this loss promotes cancer cell migration and invasion while it is associated with poor prognosis (22). Moreover, induced overexpression of LIMA1 in cancer cells appears to reverse their invasive phenotype and to reduce the metastatic potential, indicating that LIMA1 could be an excellent drug target for cancer treatment (21). In addition, immunohistochemical staining of LIMA1 in ovarian cancer showed that few cancer samples expressed the protein while downregulation of LIMA1 in ovarian cancer cells resulted in increased growth, invasion, adhesion and migration in vitro (23). Notably, LIMA1 was identified as a target of the p53 family and the downregulation of the gene can be a result of p53 mutation resulting in decreased survival of cancer patients (22).

Importin subunit $\alpha-1$ (KPNA2) was found to be significantly upregulated in cervical cancer cell lines when compared to the normal keratinocytes in our proteomic study. KPNA2, which is involved in nucleocytoplasmic trafficking, has prognostic potential in various types of cancer. Specifically, increased expression of KPNA2 has been associated with poor prognosis in astrocytic gliomas (24), gastric adenocarcinoma (25), prostate cancer (26), cholangiocarcinoma (27) and colorectal cancer (28). Furthermore, in the most recent studies, KPNA2 was suggested to be a predictive marker of gemcitabine sensitivity and survival in cholangiocarcinoma (27) and a predictor of survival following radical surgery for colorectal cancer (28).

Serum paraoxonase/arylesterase 2 (PON2) is a ubiquitously expressed cellular antioxidant and was found to be upregulated in cervical cancer in all three of our comparisons, by both proteomics and western blot analysis. PON2 has been reported to play a protective role in oral squamous cell cancer cells against irradiation-induced apoptosis (29) and higher levels of the protein are associated with a higher relapse rate in oral squamous cell cancer patients after either surgery, radiotherapy or chemotherapy (30). Importantly, the regulation of PON2-induced therapy resistance is regulated through the Wnt/glycogen synthase kinase $3 \beta$ (GSK3 $\beta) / \beta$-catenin pathway (30). Increased expression of PON2 has also been detected in bladder cancer tissues compared to that in normal tissues, and induced overexpression of the gene in bladder cancer cells led to higher cell proliferation and oxidative stress resistance (31). However, a recent study showed that PON2 can be a tumor suppressor as well (32). Particularly, PON2 expression was elevated in early stages of ovarian cancer compared to normal tissue but not in late stages of the disease. In addition, induced overexpression of PON2 in a mouse xenograft model of ovarian cancer resulted in reduced cell proliferation (32). Although the findings concerning the role of PON2 in cancers 
Table I. Shortlist of 21 proteins consistently deregulated in cervical cancer cell lines compared to normal cervical keratinocytes that are novel findings based on existing literature.

\begin{tabular}{|c|c|c|c|c|c|c|c|}
\hline \multirow[b]{2}{*}{ Symbol } & \multirow[b]{2}{*}{ Name } & \multicolumn{2}{|c|}{ HeLa vs. HCK1T } & \multicolumn{2}{|c|}{ SiHa vs. HCK1T } & \multicolumn{2}{|c|}{ C33A vs. HCK1T } \\
\hline & & $\begin{array}{l}\text { No. of } \\
\text { peptides }\end{array}$ & Ratio & $\begin{array}{c}\text { No. of } \\
\text { peptides }\end{array}$ & Ratio & $\begin{array}{l}\text { No. of } \\
\text { peptides }\end{array}$ & Ratio \\
\hline LIMA1 & LIM domain and actin-binding protein 1 & 6 & 0.20 & 7 & 0.24 & 6 & 0.09 \\
\hline KPNA2 & Importin subunit $\alpha-1$ & 9 & 124.09 & 6 & 46.84 & 13 & 351.64 \\
\hline PON2 & Serum paraoxonase/arylesterase 2 & 4 & 12.93 & 2 & 4.80 & 3 & 11.99 \\
\hline DNAJA1 & DnaJ homolog subfamily A member 1 & 4 & 7.70 & 7 & 6.70 & 6 & 7.44 \\
\hline NSUN2 & tRNA [cytosine (34)-C (5)]-methyltransferase & 8 & 6.63 & 11 & 9.60 & 7 & 4.20 \\
\hline FUS & RNA-binding protein FUS & 4 & 5.22 & 4 & 5.08 & 3 & 6.45 \\
\hline EEF1A2 & Elongation factor $1-\alpha 2$ & 12 & 5.75 & 6 & 4.03 & 11 & 5.41 \\
\hline RANGAP1 & Ran GTPase-activating protein 1 & 5 & 3.18 & 7 & 4.65 & 7 & 7.30 \\
\hline PSMB4 & Proteasome subunit $\beta$ type- 4 & 3 & 5.10 & 2 & 3.88 & 3 & 5.74 \\
\hline AHSA1 & $\begin{array}{l}\text { Activator of } 90 \mathrm{kDa} \text { heat shock protein ATPase } 1 \\
\text { homolog }\end{array}$ & 2 & 2.84 & 4 & 3.25 & 5 & 8.02 \\
\hline GMPS & GMP synthase (glutamine-hydrolyzing) & 12 & 5.73 & 6 & 2.40 & 13 & 5.93 \\
\hline PSMB7 & Proteasome subunit $\beta$ type- 7 & 3 & 4.76 & 2 & 4.36 & 3 & 4.60 \\
\hline CAPRIN1 & Caprin-1 & 4 & 4.52 & 4 & 3.70 & 4 & 4.44 \\
\hline SSRP1 & FACT complex subunit SSRP1 & 12 & 3.01 & 12 & 3.15 & 14 & 6.34 \\
\hline TOMM34 & Mitochondrial import receptor subunit TOM34 & 6 & 4.02 & 4 & 2.48 & 7 & 5.56 \\
\hline DHX9 & ATP-dependent RNA helicase A & 8 & 3.73 & 5 & 2.36 & 10 & 5.50 \\
\hline MAT2A & S-adenosylmethionine synthase isoform type-2 & 4 & 2.55 & 5 & 2.89 & 8 & 6.13 \\
\hline PABPC1 & Polyadenylate-binding protein 1 & 8 & 3.40 & 9 & 3.31 & 8 & 4.19 \\
\hline KHSRP & Far upstream element-binding protein 2 & 6 & 2.29 & 9 & 3.63 & 9 & 4.93 \\
\hline G3BP1 & Ras GTPase-activating protein-binding protein 1 & 5 & 2.65 & 5 & 2.57 & 9 & 5.39 \\
\hline ACAT1 & Acetyl-CoA acetyltransferase, mitochondrial & 6 & 2.26 & 8 & 3.32 & 8 & 3.68 \\
\hline
\end{tabular}

For all proteins, Mann-Whitney P $\leq 0.05$. Cervical cancer cell lines: HeLa (HPV18 $)$, SiHa (HPV16 $)$, C33A (HPV) and normal cervical keratinocytes (HCK1T).
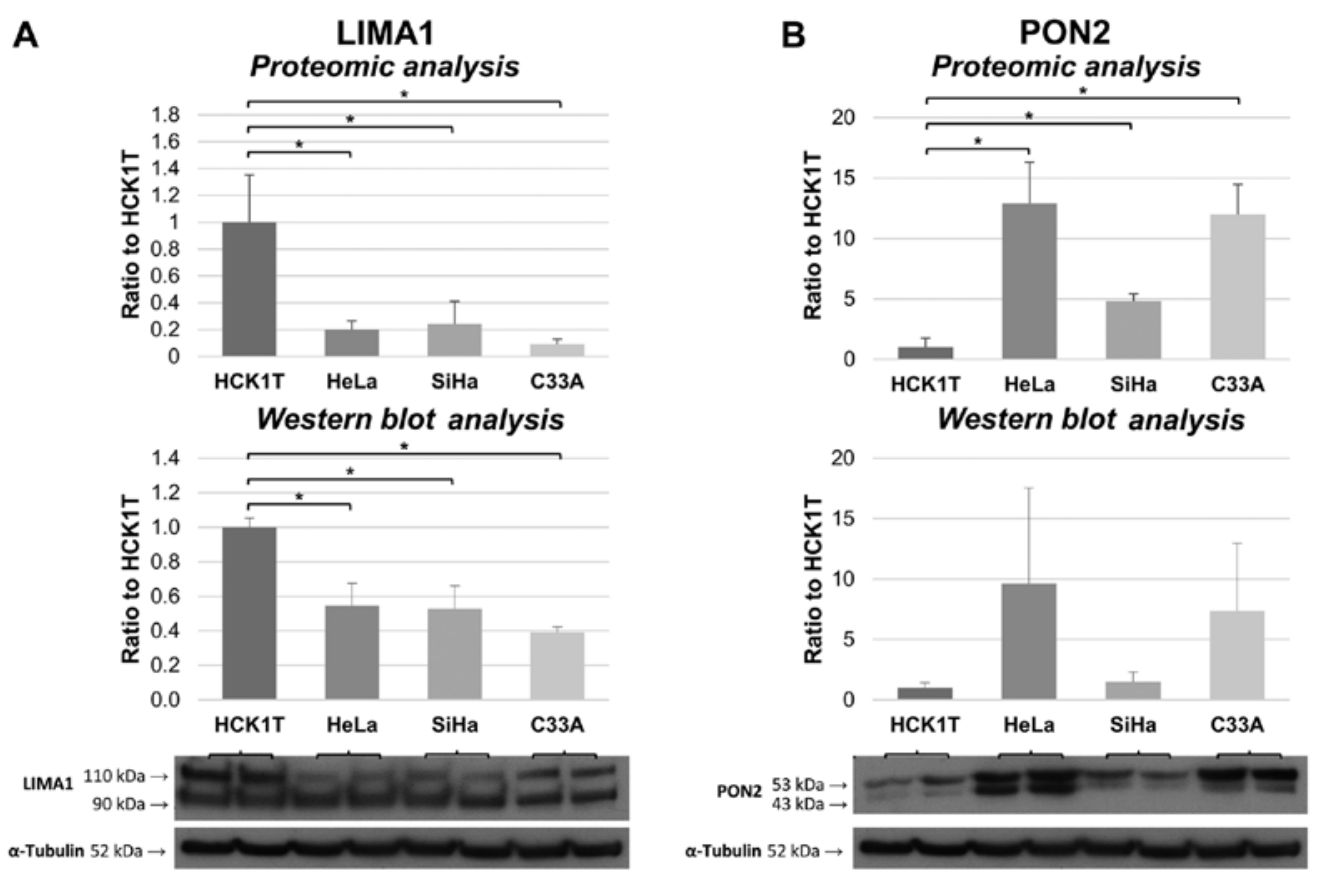

Figure 6. The proteomic expression trend of LIMA1 and PON2 is confirmed by Western blot analysis. The expression change of each protein is expressed as a ratio to the levels in HCK1T (normal keratinocytes) for both approaches. Tukey's HSD, "P $\leq 0.05$. LIMA1, LIM domain and actin-binding protein 1; PON2, serum paraoxonase/arylesterase 2. 
of different origin vary, it could certainly be a promising drug target and further investigation is warranted.

Caprin-1 (CAPRIN1) was also found to be upregulated in our analysis in cervical cancer cell lines when compared to the expression level in normal cervical keratinocytes. Caprin-1 protein is considered to enhance osteosarcoma tumor growth and lung metastasis in mice via the Akt and ERK1/2 pathways (33). CAPRIN1 was also found to be overexpressed in breast cancer cells compared to normal breast cells, and induced overexpression of the gene promoted cell proliferation and invasion while this phenotype was reversed by miRNA-223 (34). Finally, based on immunohistochemical data, CAPRIN1 expression was upregulated in hepatocellular carcinoma compared to peritumoral tissue and was significantly associated with worse hepatocellular carcinoma patient survival (35).

The high resolution proteomic analysis performed in this study, comparing three different cervical cancer cell lines to normal cervical keratinocytes, provides a comprehensive dataset of proteins deregulated in cervical cancer. The generated data provide new insights into the molecular mechanisms and key regulators of cervical carcinogenesis and can be the basis for further investigations and system biology approaches. In particular, the indicated proteins with potential critical involvement in cervical malignancy can be used for clinical validation studies and functional analyses. Overall, our research contributes to a better understanding of the underlying mechanisms important for cervical cancer and opens the way for biomarker discovery and drug target identification.

\section{Acknowledgements}

The authors wish to thank Dr Tohru Kiyono (National Cancer Centre Research Institute, Tokyo, Japan) for his generous gift of the HCK1T normal cervical cell line.

\section{Funding}

This study was funded by the European Union's European Social Fund (ESF) and Greek National Funds through the Program THALIS, under the Operational Program Education and Lifelong Learning of the National Strategic Reference Framework (NSRF), Grant no. 70-3-11830 to Kalliopi I. Pappa, and by the Oncology Program of the Central Council of Health of the Ministry of Health, Grant no. 70-3-9209 to Nicholas P. Anagnou.

\section{Availability of data and materials}

The datasets generated and analyzed during the current study are available in the Figshare repository, doi: 10.6084/m9.figshare.7121540.

\section{Authors' contributions}

KIP, JZ and NPA participated in the acquisition of funding and designed and supervised the study. KIP and VL interpreted the results and drafted the manuscript. VL performed the experiments and carried out the statistical analyses. GK performed the cell culture experiments. KV and MM performed the mass spectrometry analyses. AS and GD contributed to the interpretation of the data and drafting of the results. All authors read and approved the final manuscript and agree to be accountable for all aspects of the research in ensuring that the accuracy or integrity of any part of the work are appropriately investigated and resolved.

\section{Ethics approval and consent to participate}

Not applicable.

\section{Patient consent for publication}

Not applicable.

\section{Competing interests}

The authors declare that they have no competing interests.

\section{References}

1. Bray F, Ferlay J, Soerjomataram I, Siegel RL, Torre LA and Jemal A: Global cancer statistics 2018: GLOBOCAN estimates of incidence and mortality worldwide for 36 cancers in 185 countries. CA Cancer J Clin 68: 394-424, 2018.

2. Woodman CB, Collins SI and Young LS: The natural history of cervical HPV infection: Unresolved issues. Nat Rev Cancer 7: 11-22, 2007.

3. Dewar MA, Hall K and Perchalski J: Cervical cancer screening. Past success and future challenge. Prim Care 19: 589-606, 1992.

4. Pimple S, Mishra G and Shastri S: Global strategies for cervical cancer prevention. Curr Opin Obstet Gynecol 28: 4-10, 2016.

5. Lawrenz B, Mahajan N and Fatemi HM: The effects of cancer therapy on women's fertility: What do we know now? Future Oncol 12: 1721-1729, 2016.

6. Bentivegna E, Maulard A, Pautier P, Chargari C, Gouy S and Morice P: Fertility results and pregnancy outcomes after conservative treatment of cervical cancer: A systematic review of the literature. Fertil Steril 106: 1195-1211 e1195, 2016.

7. Barra F, Lorusso D, Leone Roberti Maggiore U, Ditto A, Bogani G, Raspagliesi $\mathrm{F}$ and Ferrero S: Investigational drugs for the treatment of cervical cancer. Expert Opin Investig Drugs 26: 389-402, 2017.

8. Kontostathi G, Zoidakis J, Anagnou NP, Pappa KI, Vlahou A and Makridakis M: Proteomics approaches in cervical cancer: Focus on the discovery of biomarkers for diagnosis and drug treatment monitoring. Expert Rev Proteomics 13: 731-745, 2016.

9. Pappa KI, Kontostathi G, Makridakis M, Lygirou V, Zoidakis J, Daskalakis G and Anagnou NP: High resolution proteomic analysis of the cervical cancer cell lines secretome documents deregulation of multiple proteases. Cancer Genomics Proteomics 14: 507-521, 2017.

10. Pappa KI, Christou P, Xholi A, Mermelekas G, Kontostathi G, Lygirou V, Makridakis M,Zoidakis J and Anagnou NP: Membrane proteomics of cervical cancer cell lines reveal insights on the process of cervical carcinogenesis. Int J Oncol 53: 2111-2122, 2018.

11. Makridakis M, Gagos S, Petrolekas A, Roubelakis MG, Bitsika V, Stravodimos K, Pavlakis K, Anagnou NP, Coleman J and Vlahou A: Chromosomal and proteome analysis of a new T24-based cell line model for aggressive bladder cancer. Proteomics 9: 287-298, 2009.

12. Narisawa-Saito M, Handa K, Yugawa T, Ohno S, Fujita M and Kiyono T: HPV16 E6-mediated stabilization of ErbB2 in neoplastic transformation of human cervical keratinocytes. Oncogene 26: 2988-2996, 2007.

13. Yugawa T, Handa K, Narisawa-Saito M, Ohno S, Fujita M and Kiyono T: Regulation of Notch1 gene expression by p53 in epithelial cells. Mol Cell Biol 27: 3732-3742, 2007.

14. Makridakis M and Vlahou A: GeLC-MS: A sample preparation method for proteomics analysis of minimal amount of tissue. Methods Mol Biol 1788: 165-175, 2018.

15. Filip S, Vougas K, Zoidakis J, Latosinska A, Mullen W, Spasovski G, Mischak H, Vlahou A and Jankowski J: Comparison of depletion strategies for the enrichment of low-abundance proteins in urine. PLoS One 10: e0133773, 2015.

16. Babicki S, Arndt D, Marcu A, Liang Y, Grant JR, Maciejewski A and Wishart DS: Heatmapper: Web-enabled heat mapping for all. Nucleic Acids Res 44: W147-W153, 2016. 
17. Bindea G, Mlecnik B, Hackl H, Charoentong P, Tosolini M, Kirilovsky A, Fridman WH, Pagès F, Trajanoski $Z$ and Galon J: ClueGO: A cytoscape plug-in to decipher functionally grouped gene ontology and pathway annotation networks. Bioinformatics 25: 1091-1093, 2009.

18. Shannon P, Markiel A, Ozier O, Baliga NS, Wang JT, Ramage D, Amin N, Schwikowski B and Ideker T: Cytoscape: A software environment for integrated models of biomolecular interaction networks. Genome Res 13: 2498-2504, 2003.

19. Giordano G, Cole TB, Furlong CE and Costa LG: Paraoxonase 2 (PON2) in the mouse central nervous system: A neuroprotective role? Toxicol Appl Pharmacol 256: 369-378, 2011.

20. Hanahan D and Weinberg RA: Hallmarks of cancer: The next generation. Cell 144: 646-674, 2011.

21. Collins RJ, Jiang WG, Hargest R, Mason MD and Sanders AJ EPLIN: A fundamental actin regulator in cancer metastasis? Cancer Metastasis Rev 34: 753-764, 2015.

22. Ohashi T, Idogawa M, Sasaki Y and Tokino T: p53 mediates the suppression of cancer cell invasion by inducing LIMA1/EPLIN Cancer Lett 390: 58-66, 2017.

23. Liu R, Martin TA, Jordan NJ, Ruge F, Ye L and Jiang WG: Epithelial protein lost in neoplasm- $\alpha($ EPLIN- $\alpha)$ is a potential prognostic marker for the progression of epithelial ovarian cancer. Int J Oncol 48: 2488-2496, 2016.

24. Gousias K, Becker AJ, Simon M and Niehusmann P: Nuclear karyopherin a2: A novel biomarker for infiltrative astrocytomas. J Neurooncol 109: 545-553, 2012.

25. Li C, Ji L, Ding ZY, Zhang QD and Huang GR: Overexpression of KPNA2 correlates with poor prognosis in patients with gastric adenocarcinoma. Tumour Biol 34: 1021-1026, 2013.

26. GruppK,Habermann M,SirmaH,SimonR, Steurer S, Hube-Magg C, Prien K, Burkhardt L, Jedrzejewska K, Salomon G, et al: High nuclear karyopherin $\alpha 2$ expression is a strong and independent predictor of biochemical recurrence in prostate cancer patients treated by radical prostatectomy. Mod Pathol 27: 96-106, 2014.

27. Tsukagoshi M, Araki K, Yokobori T, Altan B, Suzuki H, Kubo N, Watanabe A, Ishii N, Hosouchi Y, Nishiyama M, et al: Overexpression of karyopherin- $\alpha 2$ in cholangiocarcinoma correlates with poor prognosis and gemcitabine sensitivity via nuclear translocation of DNA repair proteins. Oncotarget 8 : 42159-42172, 2017.
28. Yu L, Wang G, Zhang Q, Gao L, Huang R, Chen Y, Tang Q, Liu J, Liu C, Wang $\mathrm{H}$ and Wang $\mathrm{X}$ : Karyopherin alpha 2 expression is a novel diagnostic and prognostic factor for colorectal cancer. Oncol Lett 13: 1194-1200, 2017.

29. Krüger M, Pabst AM, Al-Nawas B, Horke S and Moergel M: Paraoxonase-2 (PON2) protects oral squamous cell cancer cells against irradiation-induced apoptosis. J Cancer Res Clin Oncol 141: 1757-1766, 2015

30. Krüger M, Amort J, Wilgenbus P, Helmstädter JP, Grechowa I, Ebert J, Tenzer S, Moergel M, Witte I and Horke S: The anti-apoptotic PON2 protein is $\mathrm{Wnt} / \beta$-catenin-regulated and correlates with radiotherapy resistance in OSCC patients. Oncotarget 7: 51082-51095, 2016.

31. Bacchetti T, Sartini D, Pozzi V, Cacciamani T, Ferretti G and Emanuelli M: Exploring the role of paraoxonase-2 in bladder cancer: Analyses performed on tissue samples, urines and cell cultures. Oncotarget 8: 28785-28795, 2017.

32. Devarajan A, Su F, Grijalva V, Yalamanchi M, Yalamanchi A, Gao F, Trost H, Nwokedi J, Farias-Eisner G, Farias-Eisner R, et al: Paraoxonase 2 overexpression inhibits tumor development in a mouse model of ovarian cancer. Cell Death Dis 9: 392, 2018.

33. Sabile AA, Arlt MJ, Muff R, Husmann K, Hess D, Bertz J, Langsam B, Aemisegger C, Ziegler U, Born W and Fuchs B: Caprin-1, a novel Cyr61-interacting protein, promotes osteosarcoma tumor growth and lung metastasis in mice. Biochim Biophys Acta 1832: 1173-1182, 2013.

34. Gong B, Hu H, Chen J, Cao S, Yu J, Xue J, Chen F, Cai Y, He H and Zhang L: Caprin-1 is a novel microRNA-223 target for regulating the proliferation and invasion of human breast cancer cells. Biomed Pharmacother 67: 629-636, 2013.

35. Tan N, Dai L, Liu X, Pan G, Chen H, Huang J and Xu Q: Upregulation of caprin1 expression is associated with poor prognosis in hepatocellular carcinoma. Pathol Res Pract 213: $1563-1567,2017$ 